\title{
Guided three-dimensional molecular self-assembly on silicon substrates
}

\author{
Chia-Ching Chang ${ }^{a}$ \\ Department of Biological Science and Technology, National Chiao Tung University, Hsinchu 300, Taiwan
}

Kien Wen Sun ${ }^{\text {b) }}$

Department of Applied Chemistry, National Chiao Tung University, Hsinchu 300, Taiwan and Institute of Molecular Science, National Chiao Tung University, Hsinchu 300, Taiwan

Lou-Sing Kan

Institute of Chemistry, Academia Sinica, Taipei 115, Taiwan

Chieh-Hsiung Kuan

Department of Electrical Engineering, National Taiwan University, Taipei 106, Taiwan

(Received 2 April 2006; accepted 31 May 2006; published online 27 June 2006)

\begin{abstract}
We demonstrate three-dimensional (3D) self-assemble growth of the metallothionein (Mn,Cd-MT-2) molecules on patterned semiconductor substrates. The MT molecules deposited on the patterned substrates were found to grow into 3D rod or ring-type nanostructures, depending on the shape of patterned nanostructures on the substrates. Dense arrays of 3D molecular nanorods or rings with an area density close to $10^{10} \mathrm{~cm}^{-2}$ were demonstrated with a pore size of $20 \mathrm{~nm}$ and a pitch size of $100 \mathrm{~nm}$. Those engineered molecular nanostructures provide an excellent opportunity for biological applications, sensing sources of nanodevices, biochemical reactions on surfaces, and even single molecule studies. (C) 2006 American Institute of Physics. [DOI: 10.1063/1.2216881]
\end{abstract}

During recent years, self-assembly has become one of the most important strategies used in biology to develop complex, functional structures and to arrive at one- to threedimensional ensembles of nanostructures. Self-assembly on surfaces is one of the approaches to self-organized structures that are particularly successful. By the coordination of molecules to surfaces, the molecular systems form ordered systems-self-assembled monolayers (SAMs). Thin films of diblock copolymers were found to self-assemble into ordered periodic structures at the molecular scale $(\sim 5-50 \mathrm{~nm})$ and have been used as templates to fabricate quantum dots, ${ }^{1,2}$ nanowires, ${ }^{3-5}$ and magnetic storage media. ${ }^{6}$ More recently, in epitaxial assembly of block-copolymer films, molecular level control over the precise size, shape, and spacing of the order domains was achieved with advanced lithographic techniques. ${ }^{7}$ The development of methods for patterning and immobilizing biologically active molecules with micrometer and nanometer scale control has been proven integral to ranges of applications such as basic research, diagnostics, and drug discovery. Some of the most important advances have been in the development of biochip arrays that present either DNA, ${ }^{8}$ protein, ${ }^{9}$ or carbohydrates. ${ }^{10}$ The use of patterned substrates for components of microfluidic systems for bioanalysis is also progressing rapidly. ${ }^{11-13}$

In recent years, there have been substantial attention focused on the surface modification and patterning at the nanoscale to anchoring protein molecules on silicon substrates and reactions of organic compounds with silicon surfaces. The major attraction is the combination of the richness of organic chemistry and the versatilities of semiconductor technology. It has been demonstrated that 2,2,6,6tetramethylpiperidinyloxy (TEMPO) can bond with a single dangling bond on hydrogen-terminated $\mathrm{Si}(100)$ and $\mathrm{Si}(111)$

\footnotetext{
a) Also at the Institute of Physics, Academia Sinica, Taipei, Taiwan and the National Nano Device Laboratories, Hsinchu, Taiwan.

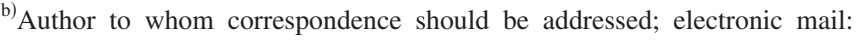
kwsun@mail.nctu.edu.tw
}

surfaces. ${ }^{14}$ Functional organic molecular layers were also found to self-assemble on metal ${ }^{15}$ and semiconductor surfaces. ${ }^{16}$ In this letter, we demonstrate methods of producing arrays of three-dimensional (3D) protein nanostructures on a Si surface. The combination of electron-beam (e-beam) lithography and self-assemble approaches provides not only a high throughput of arrays of protein nanostructures but also the highest precision.

Molecular structures of the metallothionein (MT-2) protein used in this experiment can be found in Ref. 17. The steps in synthesizing Mn,Cd-MT-2 molecules were described elsewhere. ${ }^{18}$ Spectra from both the UV absorption and the circular dichroism (CD) of our MT molecules are similar to those of the native MT-2. The effective diameter of $\mathrm{Mn}, \mathrm{Cd}-$ MT-2, measured by dynamic light scattering (DLS) spectrophotometer, is $2.86 \pm 0.29 \mathrm{~nm}$. The nanostructured templates
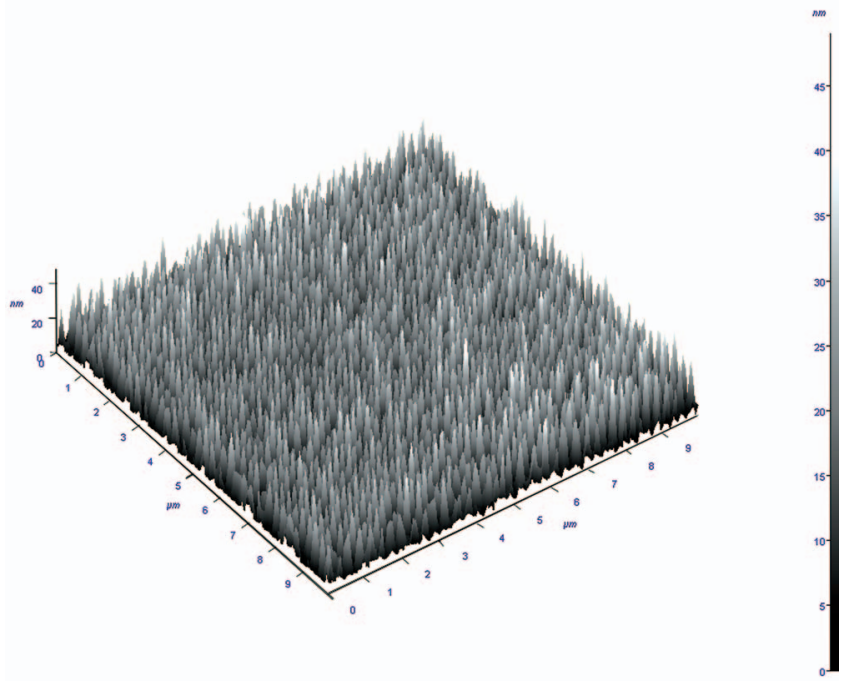

FIG. 1. (Color) AFM images of the dense molecular self-assembly arrays with a density of approximately $10^{10} \mathrm{~cm}^{-2}$. The molecules have selfassembly grown into a rod shape. 


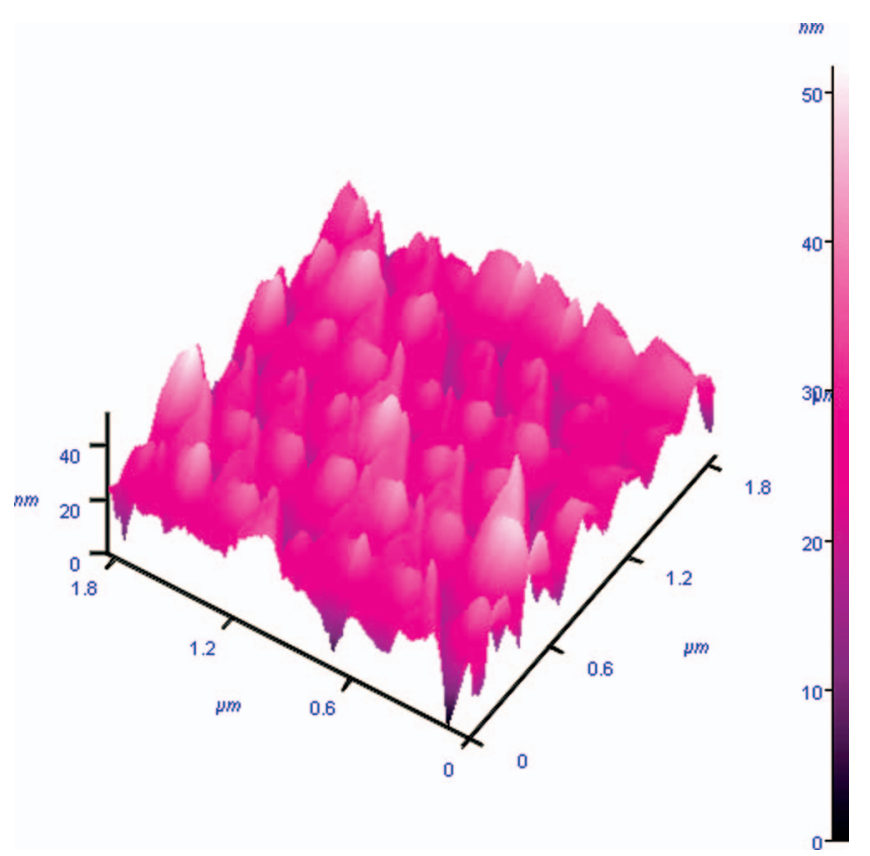

FIG. 2. (Color) Image of nanorods with AFM operated in contact mode.

were prepared on $\mathrm{Si}$ (001) substrates using e-beam lithography. The flow chart of the lithography and etching processes is given in Ref. 17 (supplementary, Fig. 2). A Si wafer was diced into $1 \mathrm{~cm}^{2}$ substrates, which were cleaned with a modified RCA solution to remove organic contaminants and then dried at $150{ }^{\circ} \mathrm{C}$ for $1 \mathrm{~h}$ to drive off excess moisture. The fabrication process commences by spinning a thin layer of ZEP-520A photoresist onto the Si wafer using a Laurell coater and prebaking it at $180{ }^{\circ} \mathrm{C}$ for $2 \mathrm{~min}$. Photoresist layers with different thicknesses were deposited onto $\mathrm{Si}$ substrates by varying the spin rates of the coater, and their thickness was determined from surface profile measurements. We were able to precisely control the photoresist thickness with accuracy within $\pm 1 \mathrm{~nm}$. Nanopore patterns were directly written in a square area of about $200 \times 200 \mu \mathrm{m}^{2}$, with an e-beam writer operated at a fixed electron voltage of $\sim 50 \mathrm{kV}$. Exposed samples were developed at $25^{\circ} \mathrm{C}$ in ZED-N50 solution for a few minutes followed by residue ZEP-520A descumed through ULVAC ozone system. By using the patterned ZEP-520 as the hard mask, the exposed $\mathrm{Si}$ area was etched into the substrate with a depth of approximately $120 \mathrm{~nm}$ via the reactive ion etching (RIE) for about $80 \mathrm{~s}$. Schematics of a completed nanostrucured template are also shown in Ref. 17 [supplementary, Fig. 2(b)]. By varying the thickness of the photoresist layers, e-beam exposure time and current, templates with nanopore sizes ranging from 20 to $150 \mathrm{~nm}$ and pitch sizes from 100 to $1200 \mathrm{~nm}$ were fabricated. Scanning electron microscope (SEM) images of the nanopores on one the templates were given in Ref. 17 (supplementary, Fig. 3). The size of the nanopores only increased by 1-2 nm after RIE processes. We were able to achieve a nanopore density as high as $10^{10} \mathrm{~cm}^{-2}$ with a pitch size of $100 \mathrm{~nm}$. The results indicated that we succeeded in preparing periodically distributed nanopore arrays on Si substrates. Their sizes and distributions are highly uniform and can be controlled with precision.

The self-assembled growth of the MT-2 proteins on the patterned templates is demonstrated as follows. The templates were immersed into the tris-HCL buffer solution with Downloaded 04 Mar 2009 to 140.112.113.225. Redistribution subject (a)

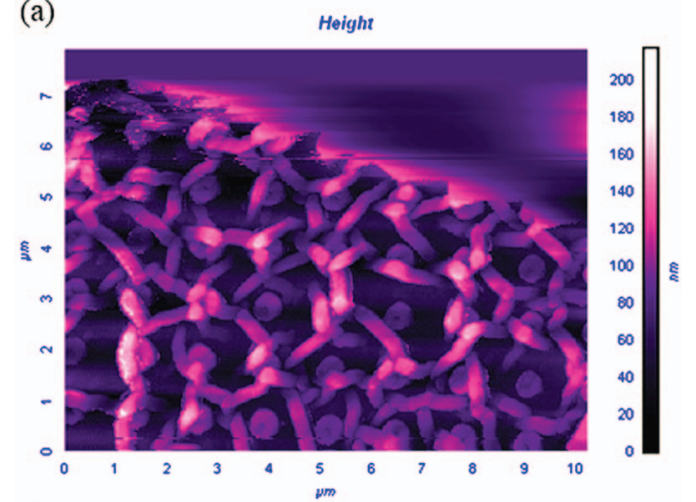

(b)

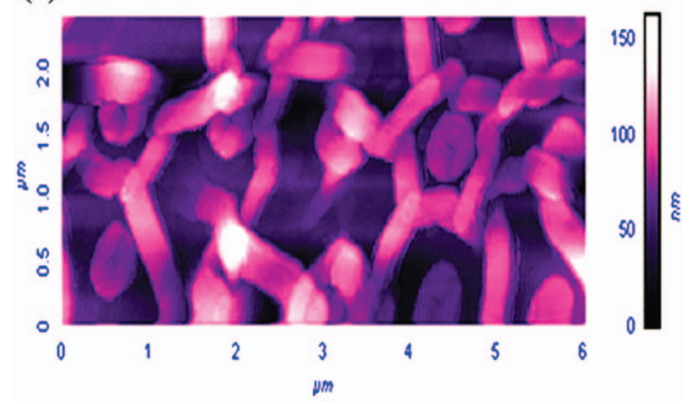

FIG. 3. (Color) (a) 3D AFM images of the molecular self-assembly on the template with unprotected Si surface. (b) Part of the image shown in (a) was enlarged by a factor of 2 .

a MT-2 concentration of $1 \mathrm{mg} / \mathrm{ml}$. An electric field, approximately $20 \mathrm{~V} / \mathrm{cm}$, was applied for $5 \mathrm{~min}$ to drive MT molecules into nanopores. The sample was then washed with de-ionized (DI) water twice to remove the unbounded MT molecules and salts on the surface (schematics of this process were shown in Ref. 17). After the deposition of MT molecules, surface profile and phase images of templates were measured with an atomic force microscope (AFM) operated in taping mode at room temperature. Figure 1 shows the image of the template surface with $40 \mathrm{~nm}$ nanopores and a pitch size of $100 \mathrm{~nm}$ after the deposition of MT molecules. Keep in mind that the top of the Si surface was still covered by photoresist after etching processes, which has prevented MT molecules to bond with the Si surface underneath. However, the surface inside nanopores inevitably contains dangling bonds after RIE processes. Therefore, the electrical field driven MT molecules were all anchored on the bare $\mathrm{Si}$ surface inside the pores. The MT molecules in each pore were found to self-assemble into a rod shape from the bottom of the pore and extended above the template surface. Those molecular nanorods have an average height of $\sim 70 \mathrm{~nm}$ above the surface with a diameter equal to the size of the pore. Dense molecular nanorod arrays, as shown in Fig. 1, were prepared on the template with a high density $\left(\sim 10^{10} \mathrm{~cm}^{-2}\right)$ of nanopores. The molecular self-assembly appears to be quite "soft" and can be manipulated with the AFM tip. This was demonstrated in the AFM image shown in Fig. 2. With the AFM operated in contact mode due to larger scanning force applied on the molecular self-assembly compared to that in the taping mode, the nanorods were all bent down and leaned along the tip scanning direction.

Experiments on templates with pore sizes larger than $100 \mathrm{~nm}$ gave quite different results. Although the MT molecules which landed inside the larger pores were able to selfassemble, molecular self-assembly was not able to extend to AIP license or copyright; see http://apl.aip.org/apl/copyright.jsp 
(a)

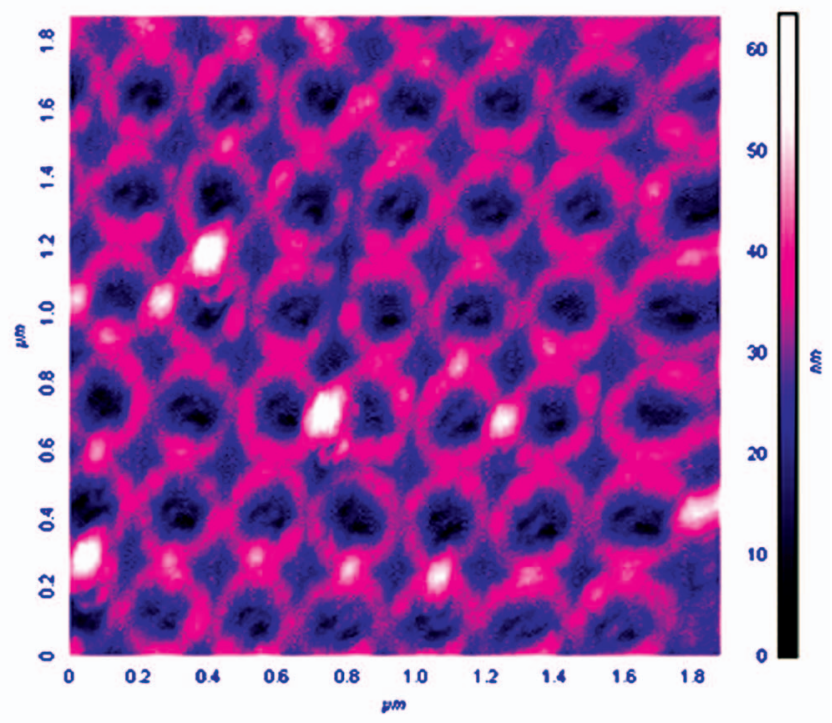

(b)

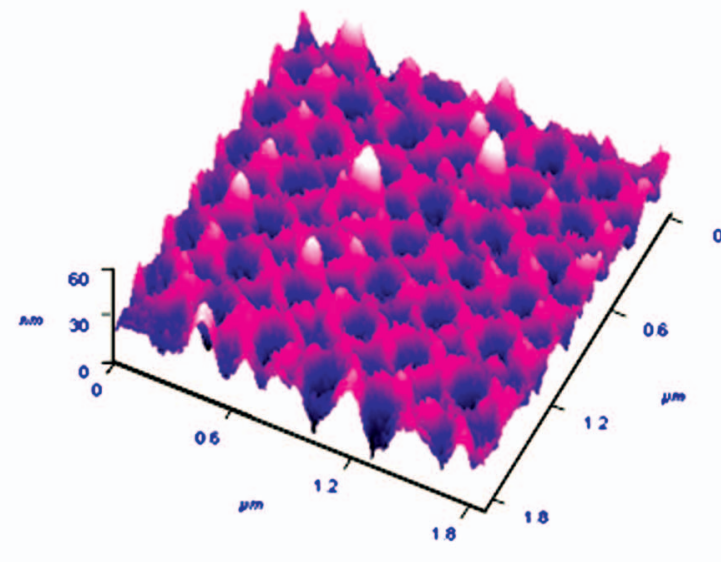

plate, the MT molecules not only self-assemble inside the pores, they also assemble along the circumference of pores to form molecular nanorings. Figure 4 shows the twodimensional (2D) and 3D AFM images of molecular rings.

It is desirable to understand the underlying selfassembled growth mechanism in order to gain better control of the formation of molecular nanostructures. The assembly of biological systems with complex structures is dictated by highly selective, noncovalent interactions, such as hydrogen bonding and van der Waals attractions. We believe that the first layer of proteins anchored inside the nanopores was bonded with the Si surface dangling bonds. They have provided building blocks for proteins which arrived at a later time. With the assistance of spatial confinement from the patterned nanostructures, the rest of the proteins are able to self-assemble via van der Waals interactions and form molecular self-assembly.

In conclusion, we have demonstrated methods of patterning molecular self-assembly and self-assembled growth of metallothionein (MT-2) molecules on nanostructured semiconductor surfaces. The MT molecules were shown to self-assemble into 3D nanostructures, depending on the structures patterned on the Si templates. Importantly, this work should not be limited to MT-2 and should be extended to other types of molecules and proteins.

This work was supported by the National Science Council of Taiwan under Contract No. NSC 94-2112-M-009-038 and by the MOE-ATU program.

${ }^{1}$ M. Park, C. Harrison, P. M. Chaikin, R. A. Register, and D. H. Adamson, Science 276, 1401 (1997).

${ }^{2}$ R. R. Li, P. D. Dapkus, M. E. Thompson, W. G. Jeong, C. Harrison, P. M. Chaikin, R. A. Register, and D. H. Adamson, Appl. Phys. Lett. 76, 1689 (2000).

${ }^{3}$ T. Thurn-Albrecht, J. Schotter, G. A. Kästle, N. Emley, T. Shibauchi, L. Krusin-Elbaum, K. Guarini, C. T. Black, M. T. Tuominen, and T. P. Russell, Science 290, 2126 (2000).

${ }^{4}$ H. C. Kim, X. Jia, C. M. Stafford, D. H. Kim, T. J. McCarthy, M. Tuominen, C. J. Hawker, and T. P. Russell, Adv. Mater. (Weinheim, Ger.) 13, 795 (2001).

${ }^{5}$ W. A. Lopes and H. M. Jaeger, Nature (London) 414, 735 (2001).

${ }^{6}$ J. Y. Cheng, C. A. Ross, V. Z.-H. Chan, E. L. Thomas, R. G. H. Lammertink, and G. J. Vancso, Adv. Mater. (Weinheim, Ger.) 13, 1174 (2001).

${ }^{7}$ S. O. Kim, H. H. Solak, M. P. Stoykovich, N. J. Ferrier, J. J. de Pablo, and P. F. Nealey, Nature (London) 424, 411 (2003).

${ }^{8}$ L. M. Demers, D. S. Ginger, S. J. Park, Z. Li, S. W. Chung, and C. A. Mirkin, Science 296, 1836 (2002).

${ }^{9}$ C. D. Hodneland, Y. S. Lee, D. H. Min, and M. Mrksich, Proc. Natl. Acad. Sci. U.S.A. 99, 5048 (2002).

${ }^{10}$ B. T. Houseman and M. Mrksich, Chem. Biol. 9, 443 (2002).

${ }^{11}$ C. S. Chen, M. Mrksich, S. Huang, G. M. Whitesides, and D. E. Ingber, Science 276, 1345 (1997)

${ }^{12}$ M. Mrksich, L. E. Dike, J. Y. Tien, D. E. Ingber, and G. M. Whitesides, Exp. Cell Res. 235, 305 (1997).

${ }^{13}$ C. S. Chen, M. Mrksich, S. Huang, G. M. Whitesides, and D. E. Ingber, Biotechnol. Prog. 14, 356 (1998).

${ }^{14}$ J. L. Pitters, P. G. Piva, X. Tong, and R. A. Wolkow, Nano Lett. 3, 1431 (2003).

${ }^{15}$ M. Fonticelli, O. Azzaroni, G. Benitez, M. E. Martins, P. Carro, and R. C. Salvarezza, J. Phys. Chem. B 108, 1898 (2004).

${ }^{16}$ B. A. Wacaser, M. J. Maughan, I. A. Mowat, T. L. Niederhauser, M. R. Linford, and R. C. Davis, Appl. Phys. Lett. 82, 808 (2003).

${ }^{17}$ See EPAPS Document No. E-APPLAB-88-204626 for the molecular structures of MT-2 proteins and preparation of Si templates. This document can be reached via a direct link in the online article's HTML reference section or via the EPAPS homepage (http://www.aip.org/pubservs/ epaps.html).

${ }^{18}$ C. C. Chang, S. F. Lee, K. W. Sun, C. C. Ho, Y. T. Chen, C. H. Chang, and L. S. Kan, Biochem. Biophys. Res. Commun. 340, 1134 (2006).

AIP license or copyright; see http://apl.aip.org/apl/copyright.jsp 